\title{
Macro-Scale Reactive Flow Model for High-Explosive Detonation in Support of ASCI Weapon Safety Milepost
}

\author{
J. E. Reaugh
}

January 3, 2002

Lawrence

Livermore

National

Laboratory

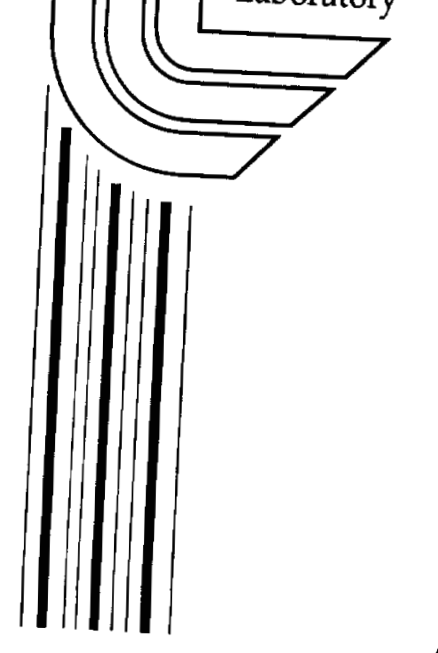




\section{DISCLAIMER}

This document was prepared as an account of work sponsored by an agency of the United States Government. Neither the United States Government nor the University of California nor any of their employees, makes any warranty, express or implied, or assumes any legal liability or responsibility for the accuracy, completeness, or usefulness of any information, apparatus, product, or process disclosed, or represents that its use would not infringe privately owned rights. Reference herein to any specific commercial product, process, or service by trade name, trademark, manufacturer, or otherwise, does not necessarily constitute or imply its endorsement, recommendation, or favoring by the United States Government or the University of California. The views and opinions of authors expressed herein do not necessarily state or reflect those of the United States Government or the University of California, and shall not be used for advertising or product endorsement purposes.

This work was performed under the auspices of the U.S. Department of Energy by the University of California, Lawrence Livermore National Laboratory under Contract No. W-7405-Eng-48.

This report has been reproduced directly from the best available copy.

Available electronically at http://www.doc.gov/bridge

Available for a processing fee to U.S. Department of Energy

And its contractors in paper from

U.S. Department of Energy

Office of Scientific and Technical Information

P.O. Box 62

Oak Ridge, TN 37831-0062

Telephone: (865) 576-8401

Facsimile: (865) 576-5728

E-mail: reports@adonis.osti.gov

Available for the sale to the public from

U.S. Department of Commerce

National Technical Information Service

5285 Port Royal Road

Springfield, VA 22161

Telephone: (800) 553-6847

Facsimile: (703) 605-6900

E-mail: orders@ntis.fedworld.gov

Online ordering: http://www.ntis.gov/ordering.htm

OR

Lawrence Livermore National Laboratory

Technical Information Department's Digital Library

http://www.llnl.gov/tid/Library.html 


\section{Introduction}

Explosive grain-scale simulations are not practical for weapon safety simulations. Indeed for nearly ideal explosives with reaction zones of order $500 \mu \mathrm{m}$, even reactive flow models are not practical for weapon safety simulations. By design, reactive flow models must resolve the reaction zone, which implies computational cells with dimension of order $50 \mu \mathrm{m}$ for such explosives.

The desired result for a simulation in which the reaction zone is not resolved is that the explosive behaves as an ideal one. The pressure at the shock front rises to the Chapman-Jouget (C)) pressure with a reaction zone dimension that is like that of a shock propagating in an unreactive medium, on the order of a few computational cells. It should propagate with the detonation velocity that is determined by the equation of state of the products. In the past, this was achieved in one dimensional simulations with "beta-burn", a method in which the extent of conversion to final product is proportional to the approach of the specific volume in the shock front to the specific volume of the CI state. One drawback with this method is that there is a relatively long build-up to steady detonation that is typically 50 to 100 computational cells. The need for relatively coarsely zoned simulations in two dimensions lead to "program-burn" by which the time to detonation can be determined by a simple ray-tracing algorithm when there are no barriers or shadows. Complications arise in two and three dimensions to the extent that some calculations of the lighting time in complex geometry can give incorrect results.

We sought to develop a model based on reactive flow that might help the needs of the Weapon Safety Simulation milepost. Important features of the model are:

1. That it be useable with any equation of state description of the explosive product gases including both JWL and LEOS table forms.

2. That it exhibits the desired dependence on zone size.

We believe that the model described here does exhibit these features.

\section{Model Description}

In the standard reactive flow model, the parameter $\lambda$ measures the extent of reaction from 0 to 1. The ignition term has the form

$$
\dot{\lambda}=A(\mu-c)^{\alpha}(1-\lambda)^{\beta}
$$

where the excess compression, $\mu$, is $\rho / \rho_{0}-1$ and the superior dot is the time derivative. We will ignore the parameter $c$ that provides a minimum excess compression needed to ignite. For our application the ignition train in our simulations are assured to result in detonation. The growth of reaction and completion of reaction both have the same form

$$
\dot{\lambda}=G \lambda^{a}(1-\lambda)^{b} p^{n}
$$

where $p$ is the pressure. In our application, the reaction is completed in the shock front, so that pressure and excess compression are tightly coupled. As a result, the forms are equivalent for us. We choose to use the ignition term.

That form can be integrated, so that

$$
v^{1-\beta}=1-(1-\beta) \int_{0}^{1} \mu^{\alpha} d t^{\prime}
$$

where $v$ is $(1-\lambda)$. At the time $\tau$, the value of $v$ is 0 , so that we can obtain a formula for $A$ 


$$
A=\frac{1}{(1-\beta) \int_{0}^{\tau} \mu^{\alpha} d t^{\prime}}
$$

To extract an approximate value of $A$, we assume that $\mu(t)$ can be approximated as

$$
\mu=\frac{\mu_{C J}}{\tau} t
$$

Then

$$
A=\frac{(1+\alpha)}{(1-\beta) \mu_{C J}^{\alpha} \tau}
$$

The value of $\tau$ is intended to be the time for passage of the detonation wave across a zone, so that

$$
\tau \approx \frac{\Delta x}{D}
$$

and

$$
A \approx \frac{(1+\alpha) D}{(1-\beta) \mu_{C J}^{\alpha} \Delta x}
$$

In this case, we have achieved the desired behavior with zone size by introducing the zone size in the parameter value, analogously to the way that similar behavior is achieved with the von Neumann artificial viscosity.

We used a simple one-dimensional test calculation to determine appropriate parameters. For fixed zone size, we determined suitable parameter values by trial and error. We determined that the results were less sensitive to changing value of $A$ when the parameter $\beta$ takes on small values and $\alpha$ is about 2. Changing the value of $A$ at a fixed zone size is equivalent to changing the zone size at a fixed value of $A$. For $1 \mathrm{~mm}$ zones the parameter $A$ ranges from 45 to 90 with satisfactory results. Evaluating Eq.5 gives a nominal value of 220 . Smaller values of $A$ result in incomplete conversion in the shock front, and so exhibit larger spike pressures. Larger values of $A$ result in a speed up of the reaction front velocity relative to CJ. For three-dimensional simulations, the effective zone size depends on the direction of wave propagation relative to the mesh. Even for cubes, the long diagonal is $73 \%$ longer than an edge. We used a onedimensional test to compare the results of a factor of 2 change in mesh size with a fixed value of the parameters (Eq. 1). We illustrate the results for LX $04 \mathrm{using} 1 \mathrm{~mm}$ and $2 \mathrm{~mm}$ zones at a location $10-\mathrm{cm}$ from the input (constant pressure) boundary. Details of the model parameters are given in Appendix 1. 


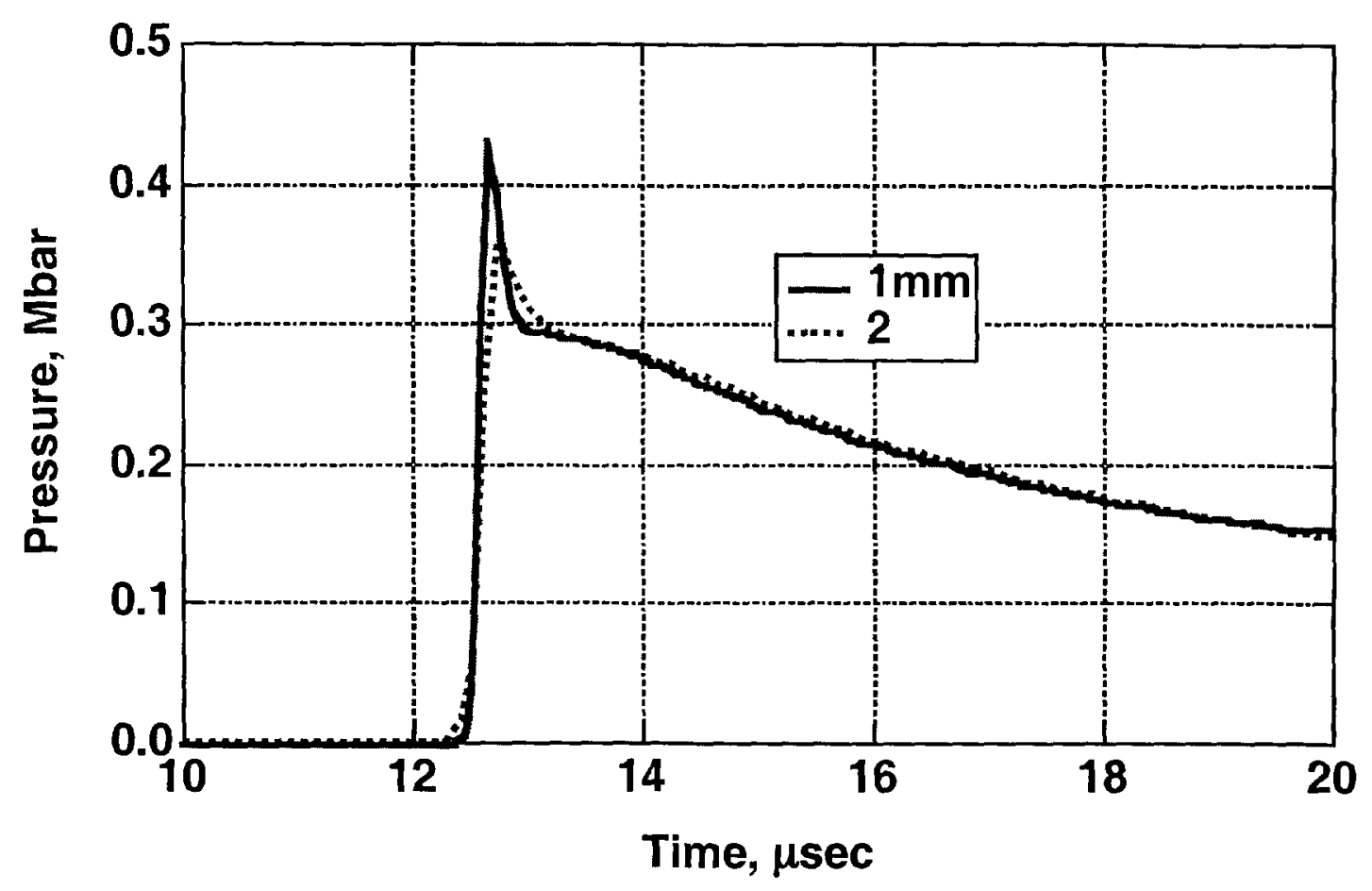

Figure 1. Pressure history $10 \mathrm{~cm}$ from input boundary for $1 \mathrm{~mm}$ and $2 \mathrm{~mm}$ zone sizes

\section{Status of tabular equations of state}

Nominal CJ conditions for LX 04 are pressure $0.34 \mathrm{Mbar}$, detonation speed $0.847 \mathrm{~cm} / \mu \mathrm{s}$. Tables constructed with CHEETAH 3.0 result in $\mathrm{CJ}$ adiabats that have $8 \%$ too much energy relative to cylinder tests, detonation speed that is $8.49 \mathrm{~km} / \mathrm{s}$, and the calculated CJ pressure is $0.418 \mathrm{Mbar}$. Tables constructed with CHEQ had $9 \%$ too much energy relative to cylinder tests, with $\mathrm{CJ}$ pressure $.33 \mathrm{Mbar}$, and detonation speed $8.3 \mathrm{~km} / \mathrm{s}$. Work is in progress to improve the CHEQ results relative to experiment, including improvements to the potential for $\mathrm{HF}$ and to the role of hydrogen bonding. The most recent results from CHEQ give detonation speed $0.84 \mathrm{~cm} / \mu \mathrm{s}$, CJ pressure 0.31 , and the energy in the cylinder test from 0 to $3 \%$ larger than reported. We will be constructing a new LEOS table with the new CHEQ values. The advantage to using an LEOS table would be improved accuracy to doubly shocked products

\section{Evaluation of the model}

We can evaluate the model in ALE 3D by comparison to program burn in geometries for which they are both appropriate. The first sample problem is a simple $10 \mathrm{~cm}$ cube with symmetry planes on each face. The mesh size is $0.133 \mathrm{~cm}$, (75 zones each direction for 420,000 total) The parameter $\operatorname{rpx}$ (Appendix 1) takes the value 50 for this mesh size. The detonation is initiated by volume-burning a $0.5-\mathrm{cm}$ radius sphere in one corner. We compare the program burn results with the new model in Figure 2 at a radius of approximately $8 \mathrm{~cm}$ along the $x$-axis, in the plane $\mathrm{z}=0$, and on the main diagonal for program burn and for the new model. The ignition volume was insufficient to ignite a beta-burn calculation, which we implemented by the use of a very low velocity program burn. The calculations were run to the same time (12 $\mu \mathrm{s}$ ) and took about the same number of cycles (225 for program burn and 280 for the new model). The computational time was significantly different. The program burn model took about 30 minutes on a SGI Octane using a single $400 \mathrm{MHz}$ processor. The new model took 245 minutes. We compare the results in Fig. 2 . The new model propagation is only $2.5 \%$ slower on 
the main diagonal than in the $x$-direction at the same radius, but it builds up to the CJ velocity. The extra delay seen in Fig. 2 is apparently a start-up problem. The initially pressurized volume has 4 -zones in the $x$-direction but only 2 on the main diagonal.

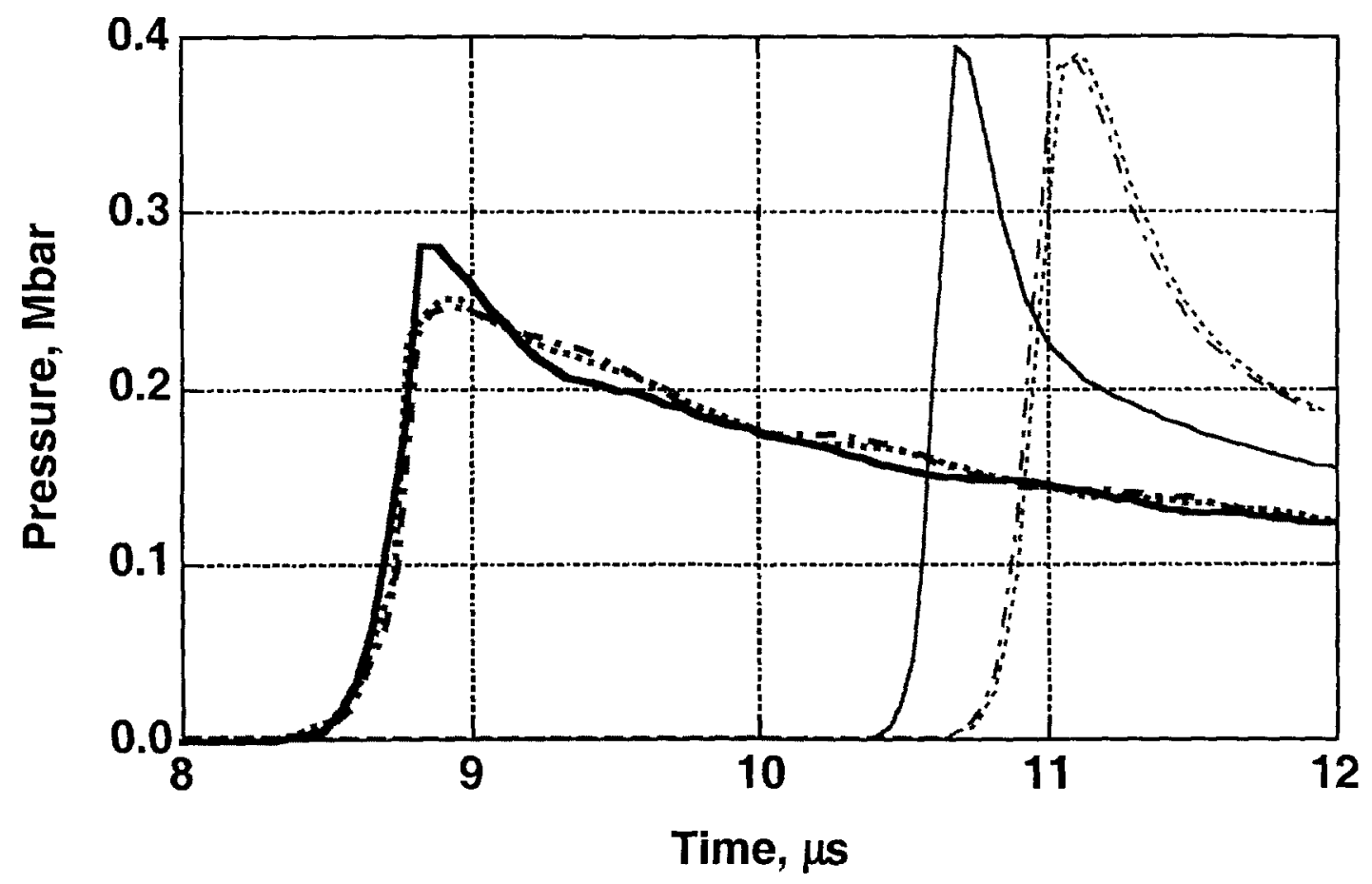

Figure 2. Pressure histories at radius about $8 \mathrm{~cm}$ at the point $(7.93,0,0)$ (solid), $(5.667,5.667,0)$ (dash-dot) and $(4.6,4.6,4.6)$ (dot) for program burn (thick) and the new model (thin). Arrival times were shifted to the radius $7.93 \mathrm{~cm}$ by using the nominal detonation velocity.

We can measure the speed of the detonation wave by calculating the arrival time at computational gauges along the $\mathrm{x}$-axis and on the main diagonal. The results are shown in Figure 3. 


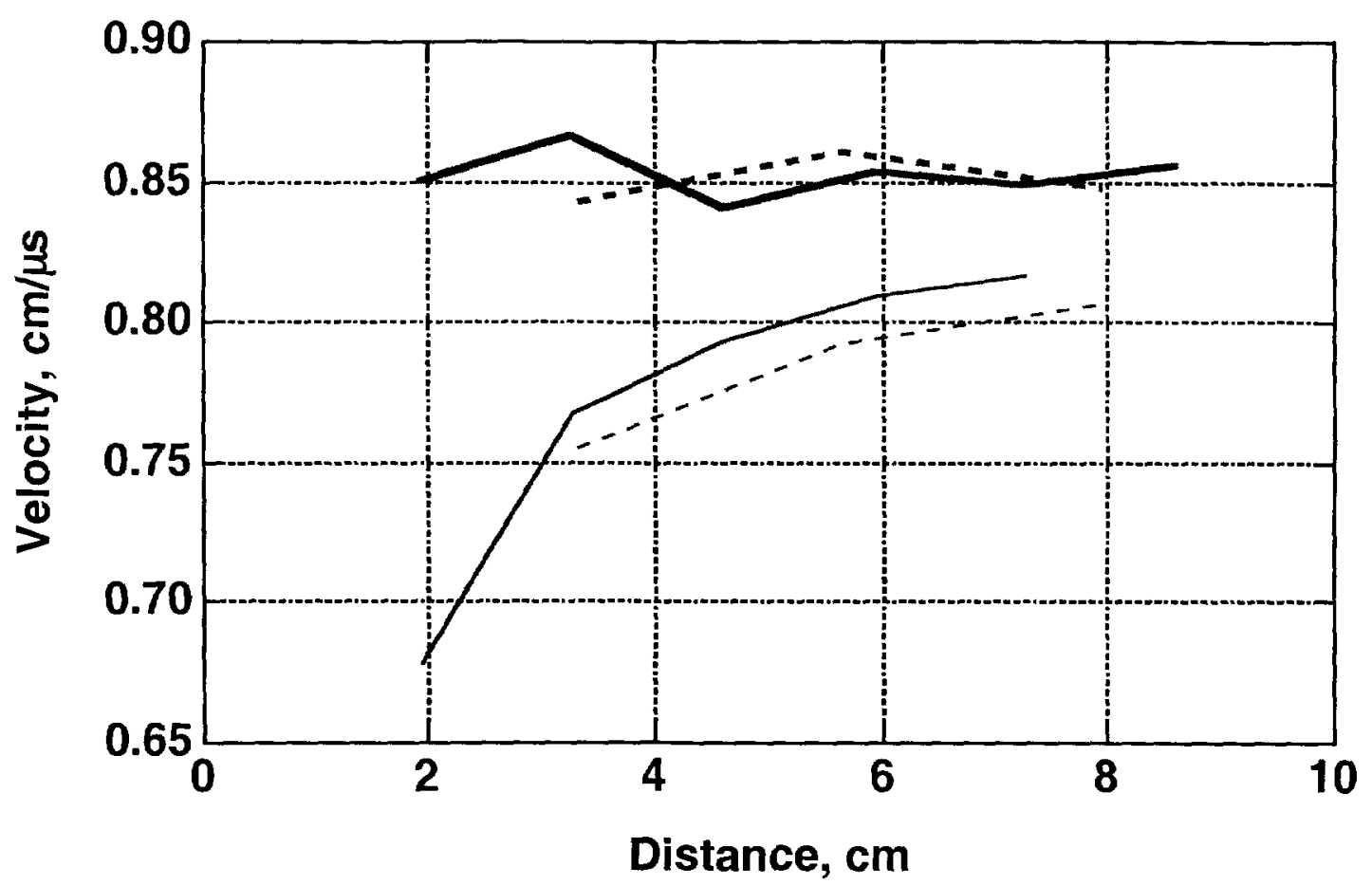

Figure 3. Detonation velocity along the x-axis (solid) and on the main diagonal (dash) for program burn (thick) and the new model (thin). Nominal CJ detonation velocity is 0.847 $\mathrm{cm} / \mu \mathrm{s}$.

The second test calculation examines the behavior of the detonation in the proximity of a free surface. By calculating in plane strain, we can examine the response of the model to a simple rarefaction. In the calculations, the 2-dimensional strip is $4 \mathrm{~cm}$ wide by $10 \mathrm{~cm}$ long. An initial thickness of $4 \mathrm{~cm}$ by $0.5 \mathrm{~cm}$ long is volume burned. This serves as a source for the new model, and was also included in the program burn model for consistency. Pressure histories along the centerline are shown in Fig. 4. Pressure contours and the mesh are shown in Figures 5 and 6. The new model shows curvature of the front that is not shown in the program burn model. The curvature is associated with incomplete reaction at the free surface. Other testing confirms that this is limited to one or two zones, independently of the zone size, so long as the parameter value is appropriately changed for the second zone size. In addition we note that the two models show complementary response in the build-up region. The new model reaches a high shock pressure quickly, although the shock speed builds up more gradually. The program burn model reaches the proper speed quickly, but builds up to peak pressure more gradually.

We show the results for geometrically scaled one-dimensional calculations in Figs. 7 and 8 . The behavior is the desired behavior

We also show a plane-strain detonation with a shadow. The explosive (red) is initiated at the top left with an initial velocity equal to the CJ particle velocity $(0.217 \mathrm{~cm} / \mu \mathrm{s})$ and is supported by a steel frame (Fig. 9). Contours of pressure at $8 \mu \mathrm{s}$ are shown in Fig. 10 using program burn with the Lund algorithm and 5 detonators at the plane $x=0$. The calculation with the new model at $9 \mu \mathrm{s}$ is shown in Fig. 11. Although the new model is later in arriving, it is at higher pressure than the program burn model. With $0.05 \mathrm{~cm}$ zones, (Fig.11) the delay is about $1 \mu \mathrm{s}$. 
We confirmed that a calculation using $0.025 \mathrm{~cm}$ zones exhibits half the delay time. For both calculations the delay time corresponds to a characteristic number of zones $(D \tau / \Delta x=13)$ in which the detonation builds up to full speed. That characteristic number depends on the initiation geometry.

\section{Summary}

We have developed a model suited to describing explosives in circumstances where the nominal zone size is larger that the reaction zone. The model is based on the existing ignition and growth models, and is implemented in ALE 3D as a chemical model that uses the ignition rate form. The behavior scales with zone size appropriately. In comparison with program burn, it does not require special coding to detonate around corners, and it can be used with any equation of state form for the reactant and for the product. The pressure waveform has a hint of a spike, so that the peak pressure is somewhat above CJ pressure very close to the initiation site. However, the rise to steady state results in a slow initial detonation speed, so that the arrival time is delayed relative to program burn. Since the run to steady state is expressed in number of zones, the delay is smaller with smaller zones.

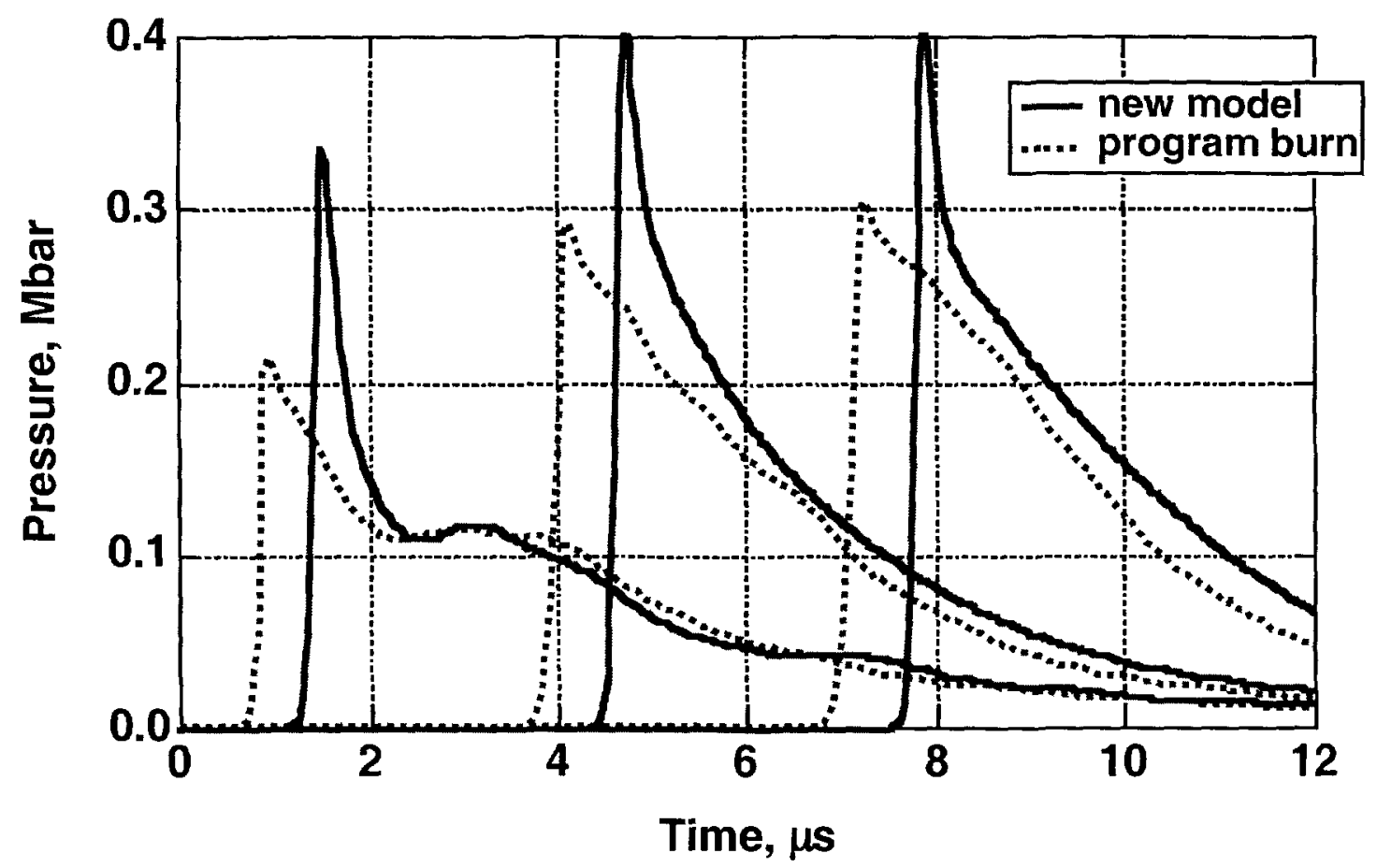

Figure 4. Pressure histories at $x$-coordinates 1,3 , and $5 \mathrm{~cm}$ on the centerline 


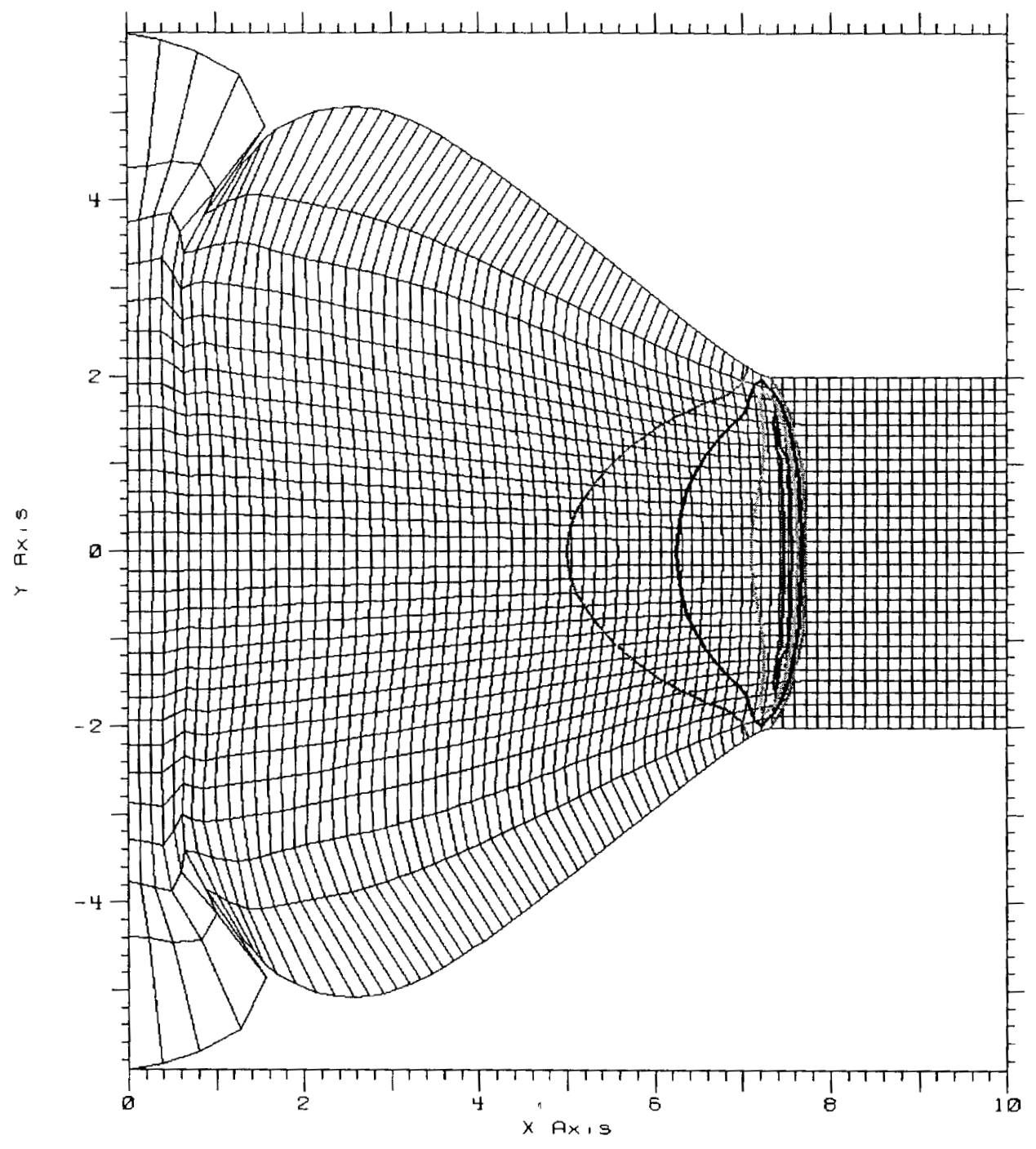

Figure 5. New model at $9 \mu \mathrm{s}$. Original strip is 4 by $10 \mathrm{~cm}$, Contour levels of pressure are 0.08 (0.08) 0.4 Mbar. 


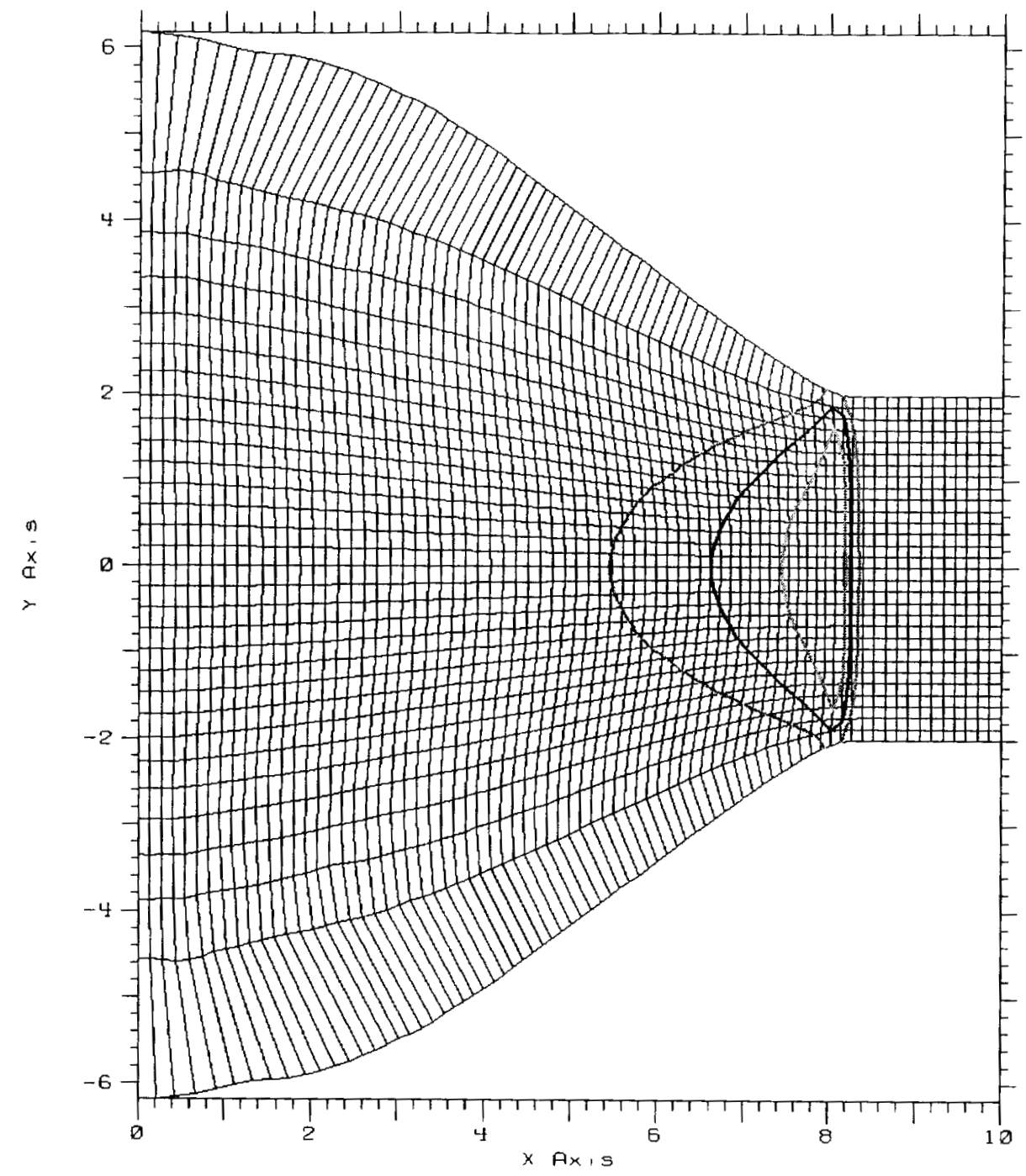

Figure 6. Program burn simulation at $6 \mu \mathrm{s}$. Original strip is 4 by $10 \mathrm{~cm}$. Contour levels of pressure are $0.08(0.08) 0.4 \mathrm{Mbar}$. 


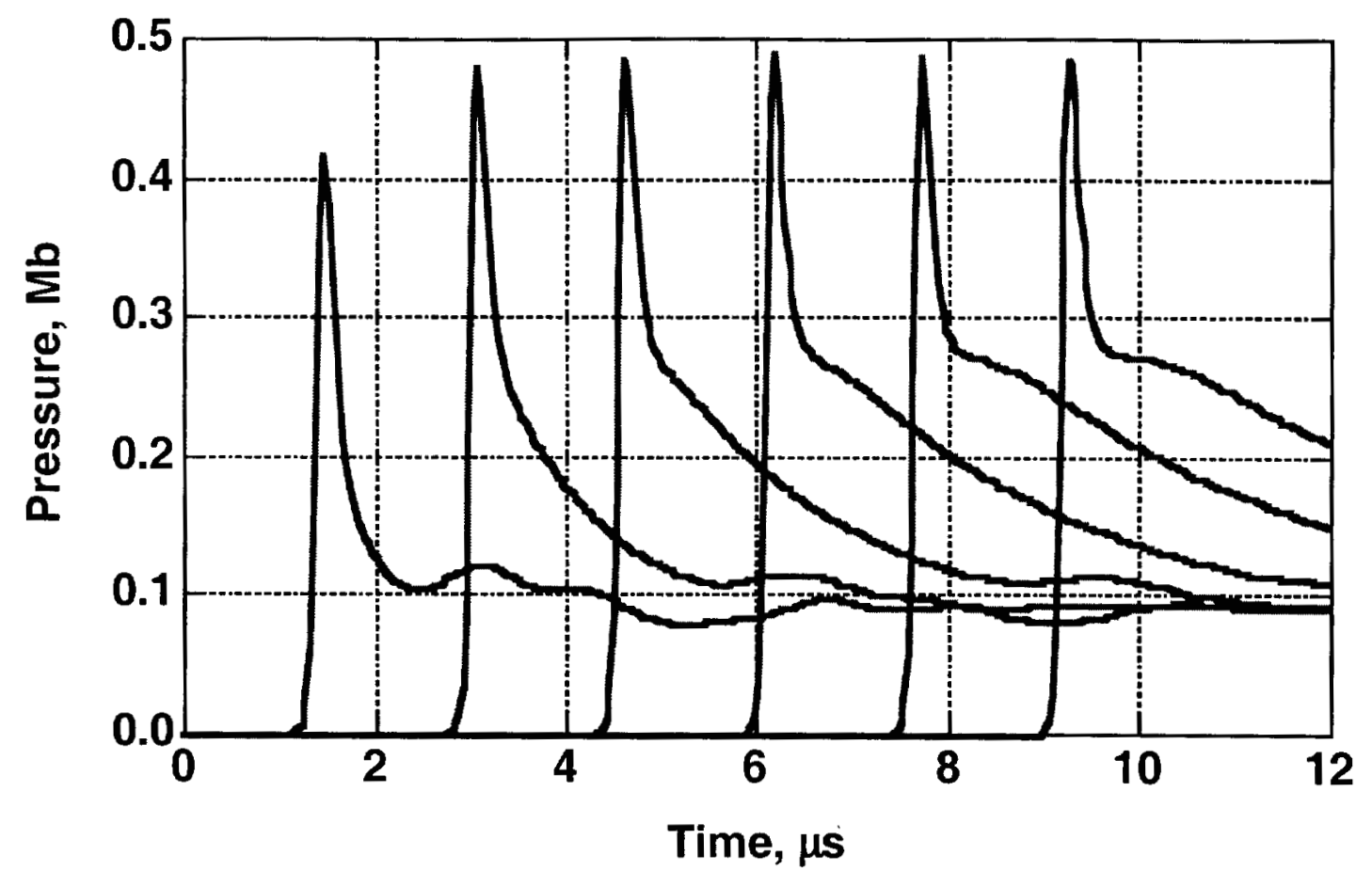

Figure 7. Pressure history at zones 10 (10) 60 for one-dimensional detonation in $10 \mathrm{~cm}$ long slab, $\Delta x=0.1333 \mathrm{~cm}, \mathrm{rpx}=50$

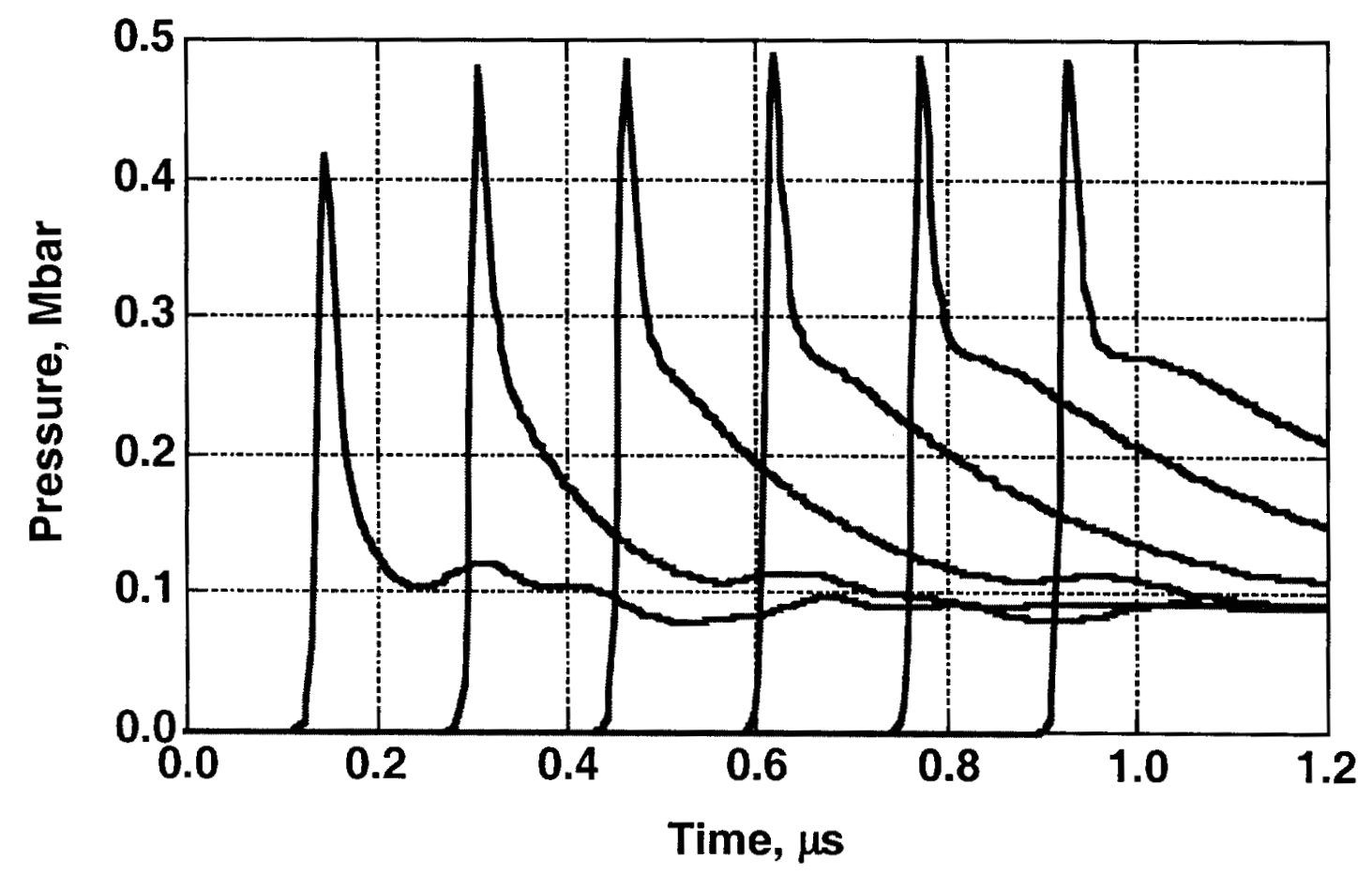

Figure 8. Pressure history at zones 10 (10) 60 for one-dimensional detonation in $1 \mathrm{~cm}$ long slab, $\Delta x=0.01333, \mathrm{rpx}=500$ 


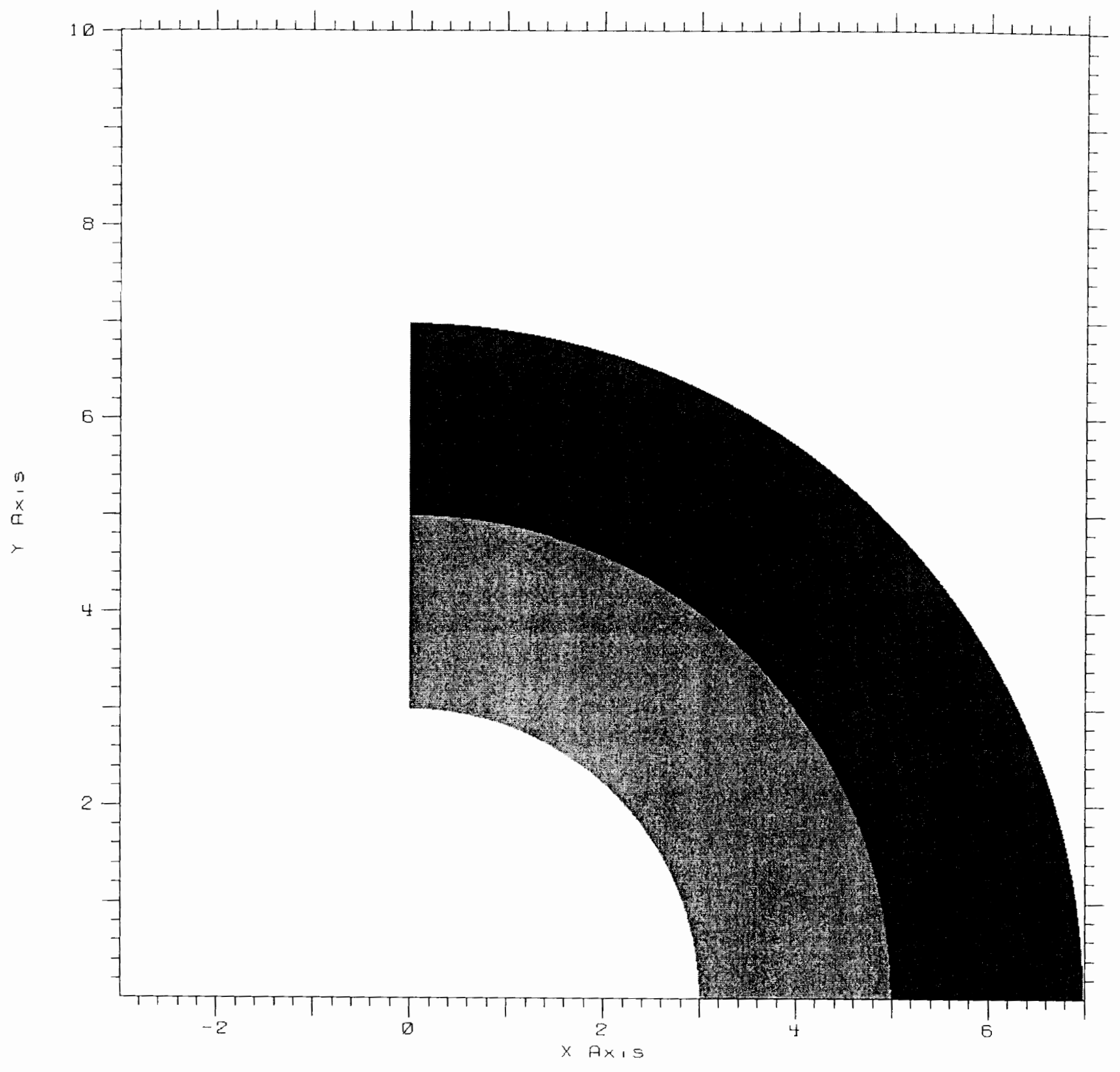

Figure 9. Explosive detonation with a shadow. Explosive is initiated at top left. The explosive wraps around the steel support. In this calculation, $\Delta x$ is approximately 0.05 . 


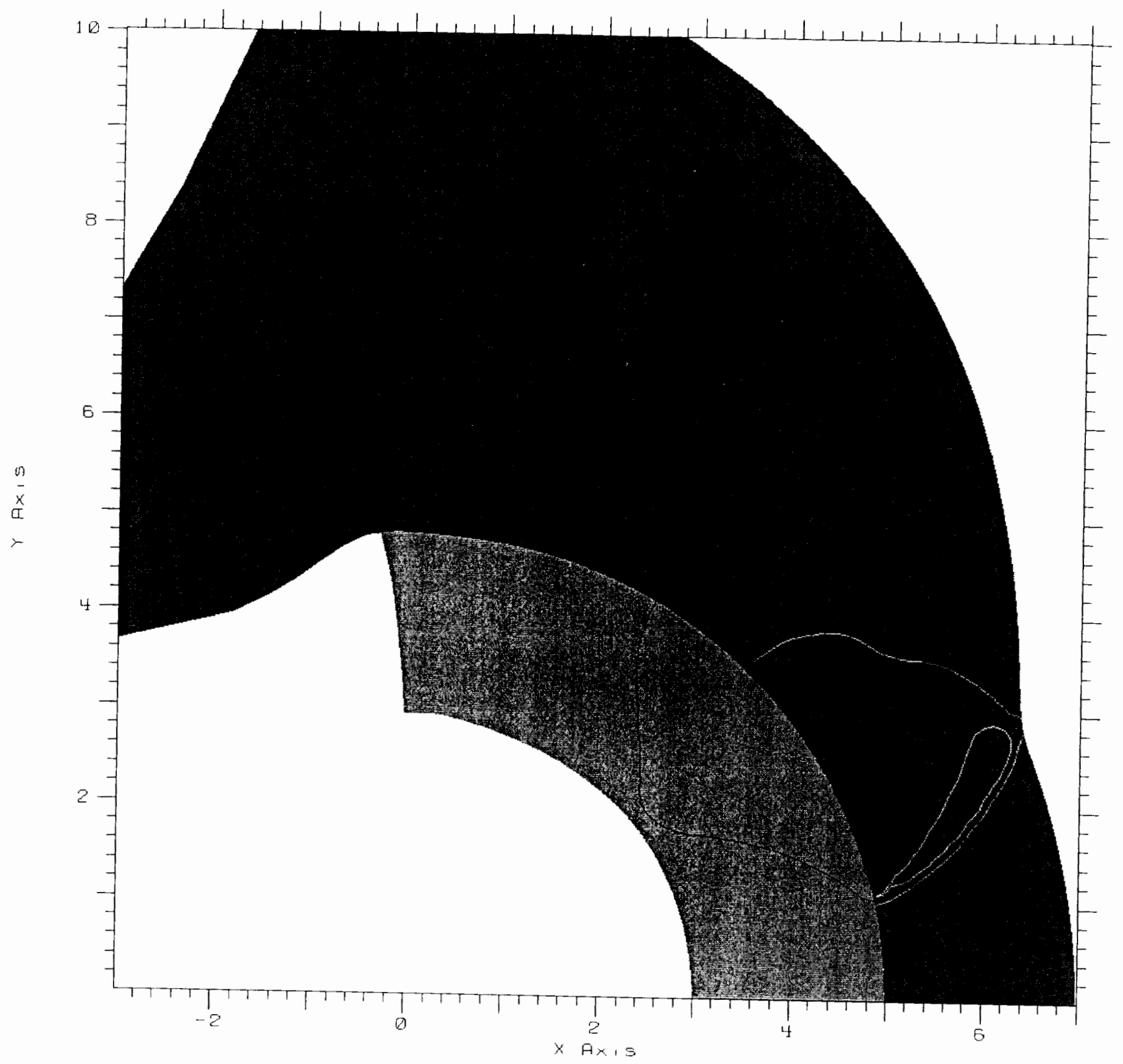

Figure 10. Program burn (Lund algorithm) at $8 \mu$ s. Contours of pressure are $0.05(0.05) 0.4$ Mbar. Peak pressure is 0.26 Mbar. 


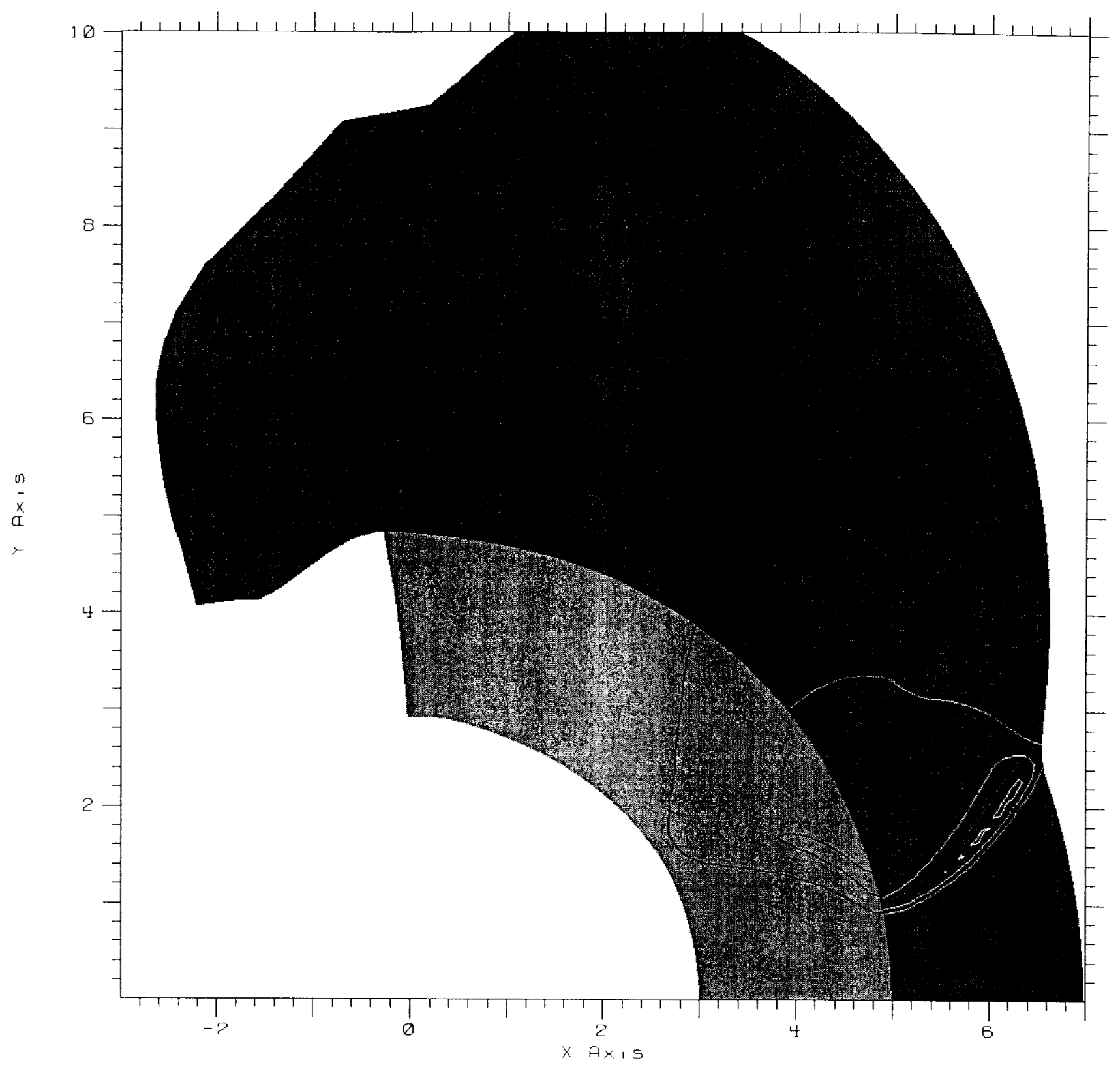

Figure 11. New model result at $9 \mu \mathrm{s}$. Contours of pressure are $0.05(0.05) 0.4 \mathrm{Mbar}$. Peak pressure is $0.33 \mathrm{Mbar}$. The parameter $\mathrm{rpx}$ is 130 . 


\section{Appendix 1. Model Parameters for LX04}

The model is implemented in ALE 3D as a compression ignition reaction in the CHEMISTRY section. For 1-mm zones, the following input fragment gives the parameter values reaction

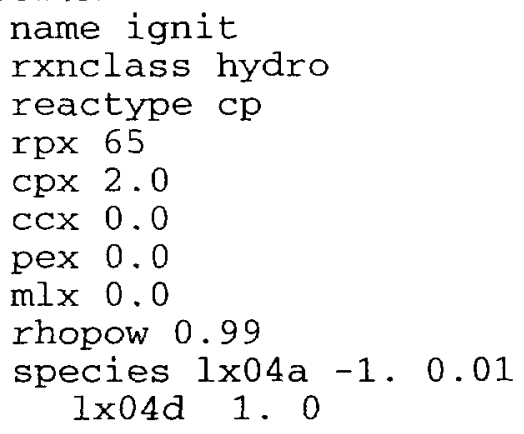

For other zone sizes, the value of rpx should be divided by the zone size in $\mathrm{mm}$.

As a chemical material, equations of state need to be included for both the reactant and the product. In trials of this model, we have used both a JWL form and LEOS tables for the product. Nominal CJ state for LX 04 is pressure $0.34 \mathrm{Mbar}$, detonation speed $0.847 \mathrm{~cm} / \mu \mathrm{s}$. At the present writing, tables constructed with CHEETAH 3.0 result in CJ adiabats that have $8 \%$ too much energy relative to cylinder tests, and detonation speed that is $8.49 \mathrm{~km} / \mathrm{s}$, although the calculated CJ pressure is $0.418 \mathrm{Mbar}$. Tables constructed with CHEQ have $9 \%$ too much energy relative to cylinder tests, but with CJ pressure $.33 \mathrm{Mbar}$, and detonation speed 8.3 $\mathrm{km} / \mathrm{s}$. Work is in progress to improve the CHEQ results relative to experiment, including improvements to the potential for $\mathrm{HF}$ and to the role of hydrogen bonding. The most recent results give detonation speed $0.84 \mathrm{~cm} / \mu \mathrm{s}$, CJ pressure 0.31 , and the energy of the cylinder test between 0 and $3 \%$ larger than reported. We will be constructing a new LEOS table with the new CHEQ values. The advantage to using an LEOS table would be improved accuracy to doubly shocked products, but should only be considered provided that the table can be made accurately enough for the CJ state.

The following input file fragment gives the JWL parameters

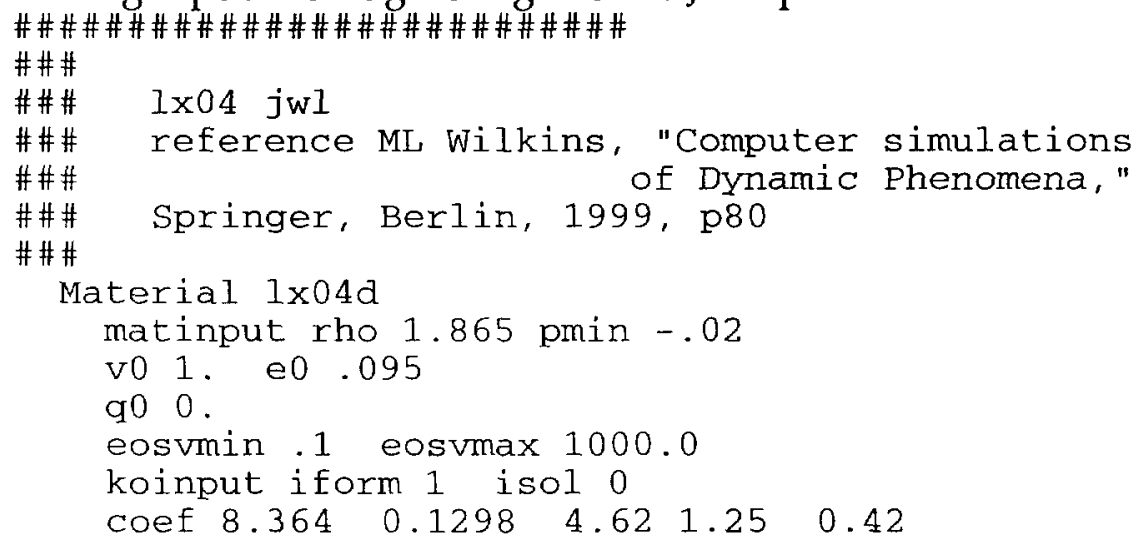

The CJ state for this JWL is $0.34 \mathrm{Mbar}, 8.47 \mathrm{~km} / \mathrm{s}$.

The reactant equation of state is constructed by a mixture rule for an appropriate mixture of HMX and Viton. The method is described in UCRL-JC-126586. The fragment of the input file that describes the reactant as a polynomial is shown below

\# ALE3D input file for $1 \times 04 a$

\#\# created Tue Dec 11 16:46:39 2001 


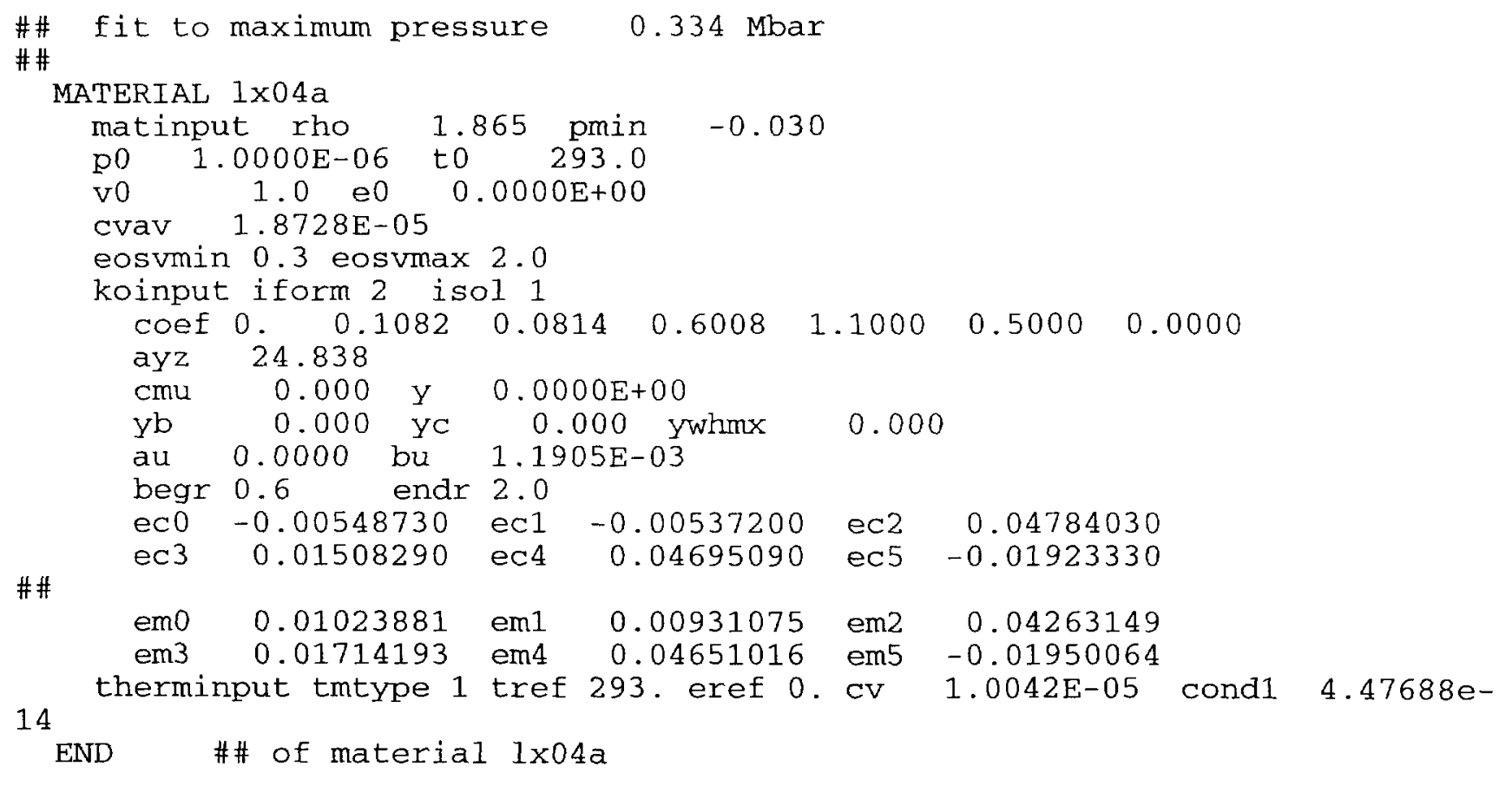




\section{Appendix 2. Input file for $10 \mathrm{~cm}$ cube}

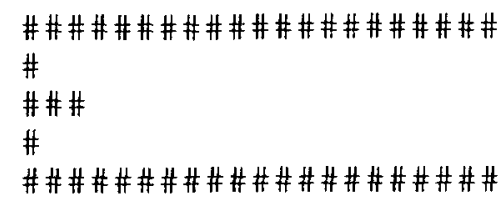

CONTROL

$$
\begin{aligned}
& \text { p_cut } 1 . e-10 \\
& \text { e_cut } 1 . e-15 \\
& \text { u_cut } 1 . e-10 \\
& \text { strsscut } 1 . e-10 \\
& \text { planetol } 1 . e-6 \\
& \text { dtmin } 2 . e-12 \\
& \text { dtmax } 1 . e 4 \\
& \text { dtinit } 0.001 \\
& \text { END \# control }
\end{aligned}
$$

HYDRO

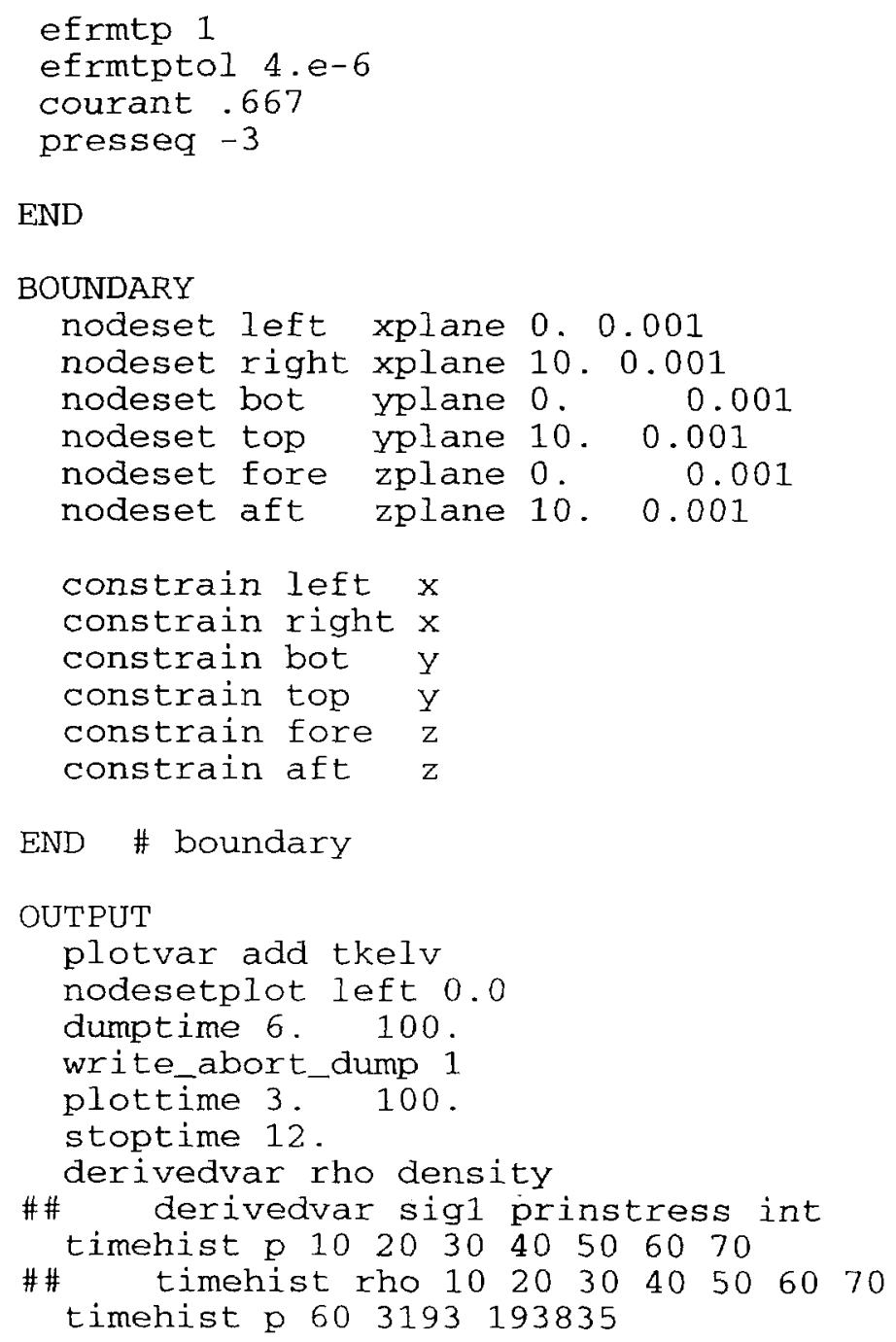




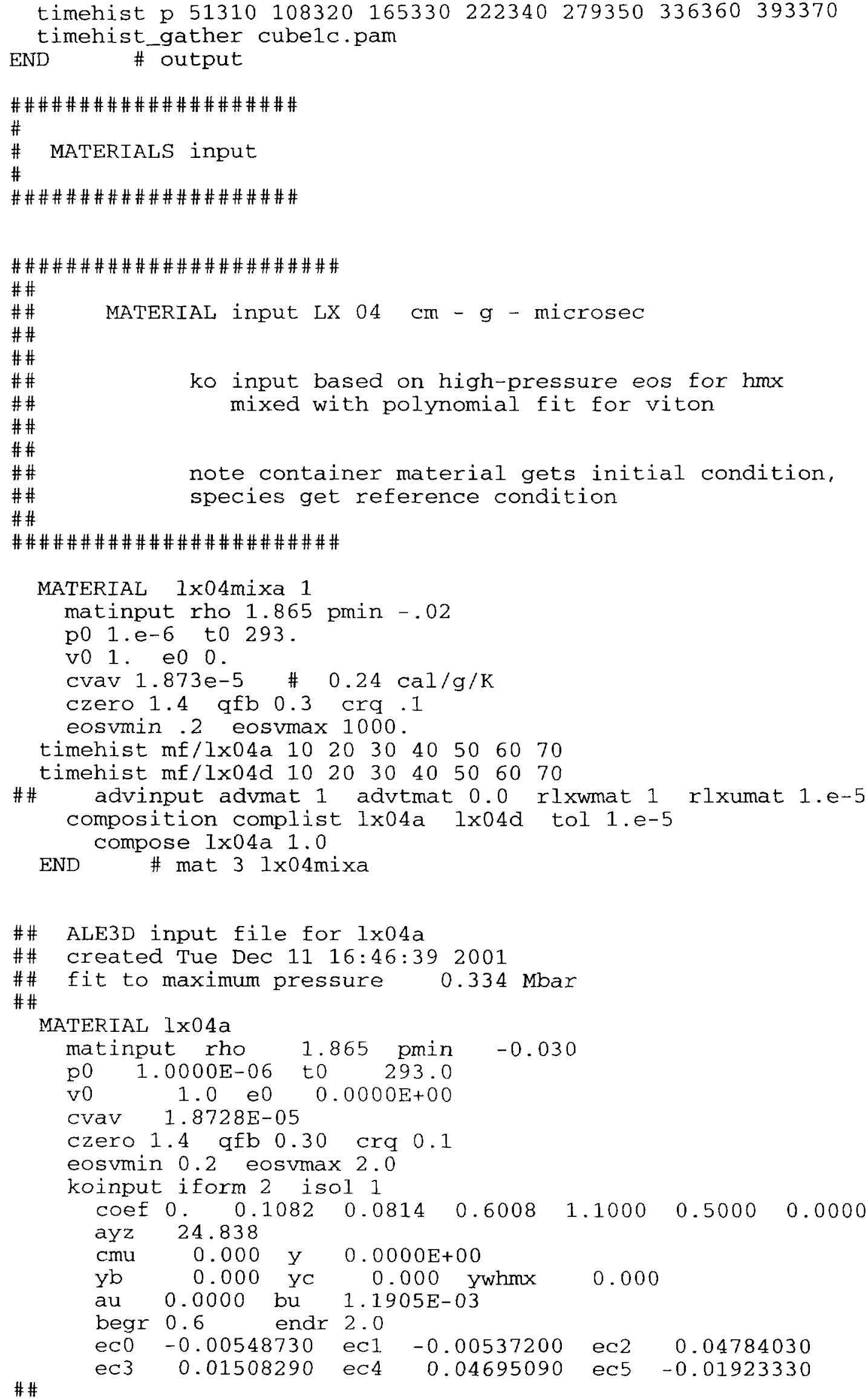




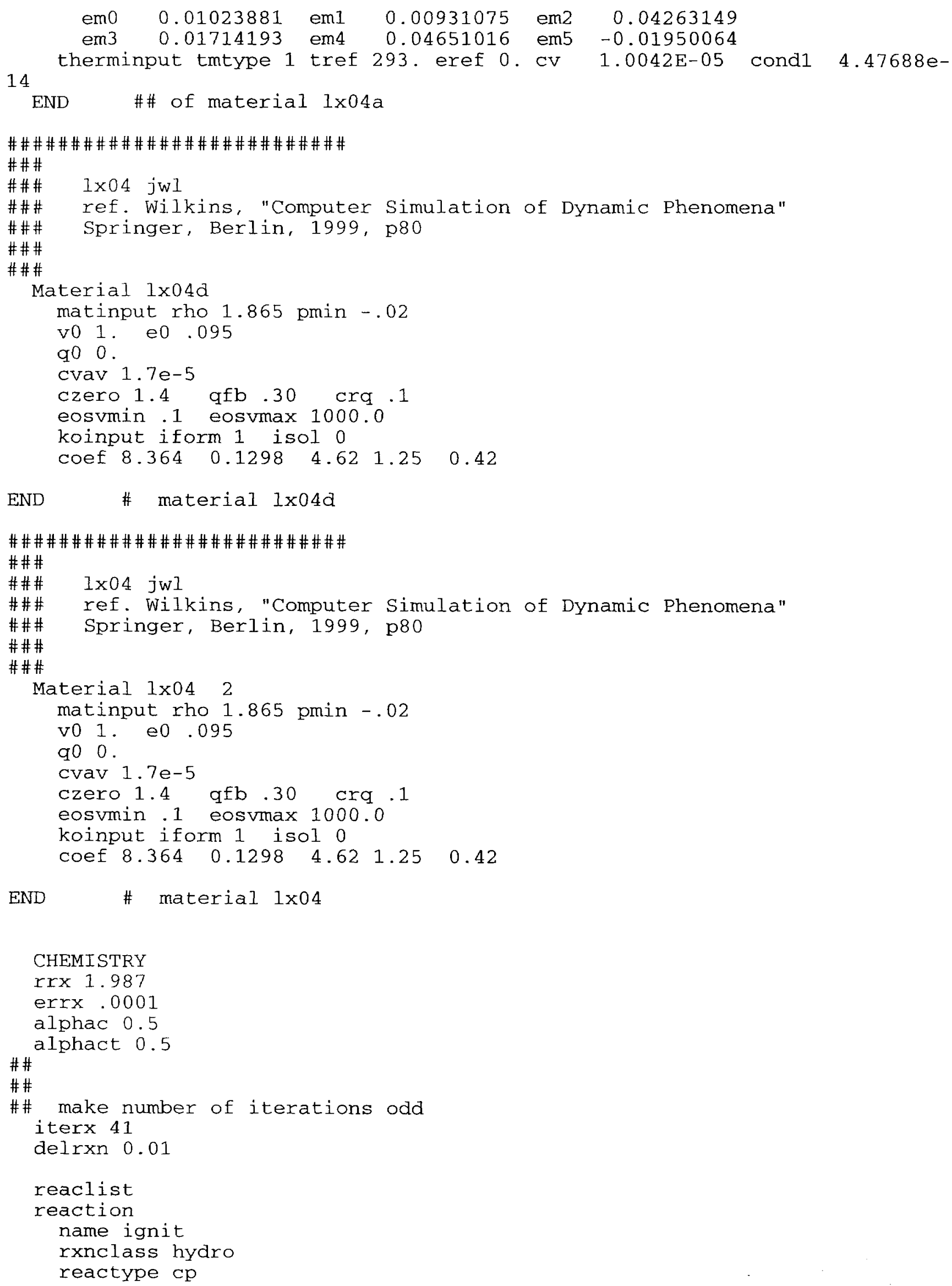




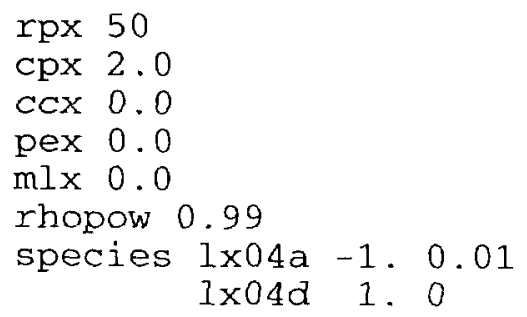

END \# chemistry

SHAPE

shap 12 END sphr 10.5000

MESH END

block $14757575 \quad 0 \quad 0 \quad 0 \quad 10.10 .10$. 